\title{
ON THE MINIMUM OF A CERTAIN INTEGRAL
}

A. SPITZBART

In this paper the following result will be proved.

Let $f(w)$ be an analytic function of $w$ for $|w|<1$, continuous for $|w| \leqq 1$, and let the value $f^{\prime}(\alpha)=1$ be prescribed at $a$ point $w=\alpha$ within the unit circle. Among functions of this type, the minimum value of the integral $\int_{C}|f(w)|^{p}|d w|$, where $p \geqq 1$ and $C$ is the unit circle $|w|=1$, is given by

$$
\phi_{1}(|\alpha|, p) \text { if } 1 \leqq p \leqq 1+|\alpha|, \quad \phi_{2}(|\alpha|, p) \text { if } p \geqq 1+|\alpha|,
$$

where

$$
\begin{aligned}
\phi_{1}(|\alpha|, p)= & 2 \pi\left(1-|\alpha|^{2}\right)^{p+1}\left[2\left(1+|\alpha|^{2}\right)\right]^{1-p} \\
& \cdot\left[(p-1)|\alpha|+\left(|\alpha|^{2}-p^{2}+2 p\right)^{1 / 2}\right]^{p-2} \\
& \cdot\left[p+|\alpha|^{2}-|\alpha|\left(|\alpha|^{2}-p^{2}+2 p\right)^{1 / 2}\right], \\
\phi_{2}(|\alpha|, p)= & 2 \pi\left(1-|\alpha|^{2}\right)^{p+1}(p-1)^{2 p-2}\left[(p-1)^{2}+|\alpha|^{2}\right]^{1-p} .
\end{aligned}
$$

These minima are attained.

As would be expected the two forms coincide if $p=1+|\alpha|$.

If $p=1$ the first form always applies and the minimum is $\phi_{1}(|\alpha|, 1)$. For $f(w)$ as in the statement of the theorem we have

$$
\begin{aligned}
\int_{C}|f(w)||d w| & \geqq \phi_{1}(|\alpha|, 1) \\
& =2 \pi\left(1-|\alpha|^{2}\right)^{2}\left[|\alpha|^{2}+\left(1+|\alpha|^{2}\right)^{1 / 2}\right]^{-1},
\end{aligned}
$$

a result which has been proved by Macintyre and Rogosinski. ${ }^{1}$

If $p \geqq 2$ the second form applies and, in particular, for $p=2$ the inequality becomes

$$
\int_{C}|f(w)|^{2}|d w| \geqq \phi_{2}(|\alpha|, 2)=2 \pi\left(1-|\alpha|^{2}\right)^{3}\left(1+|\alpha|^{2}\right)^{-1}
$$

If $\alpha=0$ the second form applies so that with $f^{\prime}(0)=1$ we have

$$
\int_{C}|f(w)| p|d w| \geqq 2 \pi
$$

We proceed to the proof. By a particularization of a result of

Presented to the Society, August 23, 1946; received by the editors December 2, 1949 and, in revised form, April 14, 1950.

1 The Edinburgh Mathematical Notes vol. 35:(1945) pp. 1-3. 
Kakeya, ${ }^{2}$ of the functions $F(z)$ which are analytic for $|z|<1$, continuous for $|z| \leqq 1$, and with the values $F(0)=A, F^{\prime}(0)=D$ assigned, the one which minimizes the integral $\int_{C^{\prime}}|F(z)| p|d z|$, with $p \geqq 1$, $C^{\prime}:|z|=1$, is given by

$$
\begin{array}{llll}
\text { (1) } & F_{0}(z)=A[p D z /(2 A)+1]^{2 / p} & \text { if } & |p D| \leqq|2 A|, \\
\text { (2) } & F_{0}(z)=-A(1-b z)^{2 / p-1}(z-b) / b & \text { if } & |p D| \geqq|2 A|,
\end{array}
$$

where

$$
\begin{aligned}
b= & -|D|\left\{|D|-\left[|D|^{2}-4|A|^{2}(2 / p-1)\right]^{1 / 2}\right\} \\
& \div[2 \bar{A} D(2 / p-1)] .
\end{aligned}
$$

We mention that the radicand appearing in $b$ is non-negative, and $|b| \leqq 1$, for $|p D| \geqq|2 A|$. If $b=0, F_{0}(z)$ in (2) is to be taken as $D z$.

The values of the minimum integrals are easily obtained and are, for the two forms (1) and (2) respectively,

$$
\begin{aligned}
& \int_{C^{\prime}}\left|F_{0}(z)\right|^{p}|d z|=2 \pi|A|^{p}\left[1+|p D /(2 A)|^{2}\right] \\
& \int_{C^{\prime}}\left|F_{0}(z)\right|^{p}|d z|=2 \pi|A|^{p}\left(1+|b|^{2}\right)|b|^{-p} .
\end{aligned}
$$

If $b$ vanishes (5) reduces to $2 \pi|D|^{p}$.

Let us now make the transformation $w=(z+\alpha) /(1+\bar{\alpha} z), \quad z$ $=(w-\alpha) /(1-\bar{\alpha} w)$. In (4) and (5) the left members become

$$
\left(1-|\alpha|^{2}\right) \int_{C}\left|F_{0}[(w-\alpha) /(1-\bar{\alpha} w)] \cdot(1-\bar{\alpha} w)^{-2 / p}\right| p|d w| .
$$

Let us set $f(w)=(1-\bar{\alpha} w)^{-2 / p} F_{0}[(w-\alpha) /(1-\bar{\alpha} w)]$, and write $f(\alpha)$ $=A^{\prime}, f^{\prime}(\alpha)=1$, which gives the relations

(6) $A=A^{\prime}\left(1-|\alpha|^{2}\right)^{2 / p}, D=\left(1-|\alpha|^{2}\right)^{2 / p}\left[p\left(1-|\alpha|^{2}\right)-2 \bar{\alpha} A^{\prime}\right] / p$.

The method of proof is to minimize $\int_{c}|f(w)|^{p}|d w|$ for each of the two forms with respect to $A^{\prime}$, and compare the values thus obtained.

The case $p=2$. We consider first the case $p=2$, for which the forms (1) and (2) coincide and become $D z+A$. We have

$$
\text { (7) } \begin{aligned}
\int_{C}|f(w)|^{2}|d w| & =2 \pi\left(1-|\alpha|^{2}\right)^{-1}\left[|A|^{2}+|D|^{2}\right] \\
& =2 \pi\left(1-|\alpha|^{2}\right)^{-1}\left[|A|^{2}+\left|\left(1-|\alpha|^{2}\right)^{2}-\bar{\alpha} A\right|^{2}\right] .
\end{aligned}
$$

2S. Kakeya, General mean modulus of analytic functions, Proceedings of the Physico-Mathematical Society of Japan (3) vol. 3 (1921) pp. 48-58. 
If $\alpha=0$ the minimum occurs for $A=0$ and is $2 \pi=\phi_{2}(0,2)$. If $\alpha \neq 0$ then for any modulus of $A$ the minimum of (7) occurs when $A$ has the same amplitude as $\alpha$, that is, if $A=k \alpha$ for some $k>0$, and (7) becomes

$$
\begin{aligned}
& 2 \pi\left(1-|\alpha|^{2}\right)^{-1}\left[|\alpha|^{2}\left(1+|\alpha|^{2}\right) k^{2}\right. \\
& \left.\quad-2|\alpha|^{2}\left(1-|\alpha|^{2}\right)^{2} k+\left(1-|\alpha|^{2}\right)^{4}\right] .
\end{aligned}
$$

The derivative with respect to $k$ vanishes for $k=\left(1-|\alpha|^{2}\right)^{2}(1$ $\left.+|\alpha|^{2}\right)^{-1}$, which yields as the minimum the value $2 \pi\left(1-|\alpha|^{2}\right)^{3}(1$ $\left.+|\alpha|^{2}\right)^{-1}=\phi_{2}(|\alpha|, 2)$.

Henceforth we exclude the value $p=2$.

The first form of $f(w)$. For $f(w)$ corresponding to $F_{0}(z)$ of the first form we have, with (4) and (6),

$$
\text { (8) } \begin{aligned}
\int C & |f(w)| p|d w| \\
& =2 \pi\left(1-|\alpha|^{2}\right)\left|A^{\prime}\right|^{p}\left[1+\left|p\left(1-|\alpha|^{2}\right) /\left(2 A^{\prime}\right)-\bar{\alpha}\right|^{2}\right],
\end{aligned}
$$

and for our problem this is to be minimized with respect to $A^{\prime}$. The condition $|p D| \leqq|2 A|$, which shall define the term admissible for the first form, becomes $\left|p\left(1-|\alpha|^{2}\right) /\left(2 A^{\prime}\right)-\bar{\alpha}\right| \leqq 1$, which excludes for the first form the possibility that $A^{\prime}=0$. If $\alpha=0$ the minimum of (8) subject to the condition on $A^{\prime}$ occurs for $\left|A^{\prime}\right|=p / 2$, and is $\phi_{3}(0, p)$, where

$$
\phi_{3}(|\alpha|, p)=2 \pi \cdot 2^{1-p} p^{p}\left(1-|\alpha|^{2}\right)(1-|\alpha|)^{p} .
$$

If $\alpha \neq 0$, for any given modulus of $A^{\prime}$ the minimum of (8) occurs when $A^{\prime}$ is a positive multiple of $\alpha$, that is, if $A^{\prime}=k^{\prime} \alpha$ for some $k^{\prime}>0$. Let us set $a=p\left(1-|\alpha|^{2}\right) /\left(2|\alpha|^{2}\right)$; the right member of (8) becomes, apart from a constant factor,

$$
k^{p}\left(1+|\alpha|^{2}\right)-2 a|\alpha|^{2} k^{p-1}+a^{2}|\alpha|^{2} k^{\prime p-2},
$$

and the condition on $A^{\prime}$ becomes $k^{\prime} \geqq k_{1}^{\prime}=p(1-|\alpha|) /(2|\alpha|)$.

If $p>1+\left(1+|\alpha|^{2}\right)^{1 / 2}$ the derivative of (10) with respect to $k^{\prime}$ vanishes for no positive value of $k^{\prime}$.

If $p \leqq 1+\left(1+|\alpha|^{2}\right)^{1 / 2}$ the derivative of (10) vanishes for

$$
\begin{aligned}
k^{\prime}=k_{0}^{\prime}=(1 & \left.-|\alpha|^{2}\right)\left[(p-1)|\alpha|+\left(|\alpha|^{2}-p^{2}+2 p\right)^{1 / 2}\right] \\
& \div\left[2|\alpha|\left(1+|\alpha|^{2}\right)\right] .
\end{aligned}
$$

We are concerned here with the relation of magnitude of $k_{1}{ }^{\prime}$ and $k_{0}^{\prime}$. If $1 \leqq p \leqq 1+|\alpha|$ only the positive root in $k_{0}^{\prime}$ gives a positive $k_{0}^{\prime}$, and we may show that $k_{0}^{\prime} \geqq k_{1}^{\prime}$, so that the minimum of (10) occurs 
for $k^{\prime}=k_{0}^{\prime}$ and the minimum of $(8)$ is $\phi_{1}(|\alpha|, p)$, which is a relative minimum.

Now suppose $p>1+|\alpha|$, so that $k_{0}^{\prime}<k_{1}^{\prime}$, and $k_{0}^{\prime}$ is not an admissible value of $k^{\prime}$. Let us write

$$
A^{\prime}=k^{\prime}|\alpha| e^{i \theta}, \quad \alpha=|\alpha| e^{i \theta_{0}} .
$$

Then (8) is a function of $k^{\prime}$ and $\theta$. For any value $k^{\prime}>0$ the minimum of (8) occurs for $\theta=\theta_{0}$, the maximum occurs for $\theta=\theta_{0}+\pi$. If we let $t=e^{i\left(\theta-\theta_{0}\right)}+e^{-i\left(\theta-\theta_{0}\right)}$, the value in (8) becomes, apart from a constant factor,

$$
\begin{gathered}
4|\alpha|^{2}\left(1+|\alpha|^{2}\right) k^{p}-2 p|\alpha|^{2} t\left(1-|\alpha|^{2}\right) k^{p-1} \\
+p^{2}\left(1-|\alpha|^{2}\right)^{2} k^{p-2}
\end{gathered}
$$

and the derivative of (12) with respect to $k^{\prime}$ is

$$
\begin{gathered}
p k^{p-3}\left[4|\alpha|^{2}\left(1+|\alpha|^{2}\right) k^{\prime 2}-2|\alpha|^{2}(p-1) t\left(1-|\alpha|^{2}\right) k^{\prime}\right. \\
\left.+p(p-2)\left(1-|\alpha|^{2}\right)\right]
\end{gathered}
$$

with the values of $t$ between -2 and 2 .

If $1+|\alpha|<p<2$, for each value of $t$ there is one positive zero of (13). These zeros give the minima of (12) with respect to $k^{\prime}$ for the different values of $\theta$. The relative minimum of these minima occurs for $t=2$, or $\theta=\theta_{0}$, and is not admissible; hence the admissible minimum, if any, occurs where

$$
\left|p\left(1-|\alpha|^{2}\right) /\left(2 A^{\prime}\right)-\bar{\alpha}\right|=1 \text {. }
$$

If none of these minima is admissible, the admissible minimum of (12) certainly occurs where (14) holds. In this event (8) reduces to $2 \pi$ $\cdot 2\left(1-|\alpha|^{2}\right)\left|A^{\prime}\right|^{p}$ for which the minimum subject to (14) occurs for $A^{\prime}=k_{1}^{\prime} \alpha$, and the minimum of $(8)$ is $\phi_{3}(|\alpha|, p)$.

If $p>2$ we have the following situation. For $t \leqq 0$ there is no positive zero of (13), and (12) increases with respect to $k^{\prime}$. For $t>0$ there are no positive zeros if

$$
p>1+2\left(1+|\alpha|^{2}\right)^{1 / 2}\left[4\left(1+|\alpha|^{2}\right)-t^{2}|\alpha|^{2}\right]^{-1 / 2},
$$

and two positive zeros if this inequality is reversed. The larger of these zeros gives the relative minimum for a fixed $t>0$. Again the minimum of these minima occurs for $\theta=\theta_{0}$ and is not admissible. The admissible minimum again occurs where (14) holds, and gives $A^{\prime}$ and $\phi_{3}(|\alpha|, p)$ as above.

For $f(w)$ corresponding to the first form of $F_{0}(z)$ the result is therefore that the minimum is $\phi_{1}(|\alpha|, p)$ if $1 \leqq p \leqq 1+|\alpha|$, and is $\phi_{3}(|\alpha|, p)$ 
if $1+|\alpha|<p<2$, or $p>2$.

The second form of $f(w)$. With (5) and (6) we have for the second form of $f(w)$.

$$
\int_{\mathrm{C}}|f(w)|^{p}|d w|=2 \pi|A|^{p}\left(1+|b|^{2}\right)\left[\left(1-|\alpha|^{2}\right)|b|^{p}\right]^{-1},
$$

with $b, A, D$ as at the start of the proof. We shall mean by admissible for the second form that the condition $|p D| \geqq|2 A|,|b| \leqq 1$ is satisfied.

We consider first the case $1 \leqq p<2$. Let us set

$$
R=|D|-\left[|D|^{2}-4|A|^{2}(2 / p-1)\right]^{1 / 2} \quad(0 \leqq R \leqq|D|) .
$$

Then

$$
\begin{aligned}
|A|^{2} & =\left(2 R|D|-R^{2}\right)[2(2 / p-1)]^{-1} \\
|b| & =R[2|A|(2 / p-1)]^{-1}
\end{aligned}
$$

and (15) becomes

$$
\begin{aligned}
2 \pi \cdot 2^{1-p}\left[\left(1-|\alpha|^{2}\right)(2-p)\right]^{-1}(2|D|-R)^{p-1} \\
\cdot[|D|(2-p)-R(1-p)] .
\end{aligned}
$$

Although $R=0$ is initially exceptional, (17) is valid also for $R=0$.

If $\alpha=0$ and $p=1,(17)$ has the value $2 \pi$. If $\alpha=0$ and $1<p<2$, (17) is valid and is a function of $|A|$ alone, since $D=1$. Its derivative with respect to $|A|$ vanishes only for $A=0$, in which case the value of (17) is again $2 \pi$. Hence if $\alpha=0$ and $1 \leqq p<2$, the minimum of (17) is $2 \pi$.

If $\alpha \neq 0$ let us again set

$$
A=k|\alpha| e^{i \theta}, \quad \alpha=|\alpha| e^{i \theta_{0}} .
$$

The expression in (17) is a function of $k$ and $\theta$. If $k=0$ the value of (17) is constant. For fixed $k>0$ the derivative with respect to $\theta$ of the part of (17) involving $k$ and $\theta$ becomes

$$
p(2-p)(2|D|-R)^{p-1} \partial|D| / \partial \theta \text {. }
$$

Now

$$
\begin{aligned}
|D|^{2}= & p^{-2}\left[p^{2}\left(1-|\alpha|^{2}\right)^{4 / p+2}\right. \\
& \left.-2 p\left(1-|\alpha|^{2}\right)^{2 / p+1}|\alpha|^{2} k\left(e^{i\left(\theta-\theta_{0}\right)}+e^{-i\left(\theta-\theta_{0}\right)}\right)+4|\alpha|^{4} k^{2}\right]
\end{aligned}
$$

so that

$2|D| \cdot \partial|D| / \partial \theta=-2 i p^{-1}\left(1-|\alpha|^{2}\right)^{2 / p+1}|\alpha|^{2} k\left(e^{i\left(\theta-\theta_{0}\right)}-e^{-i\left(\theta-\theta_{0}\right)}\right)$. 
Hence (18) vanishes only if $\theta=\theta_{0}$ or $\theta=\theta_{0}+\pi$ (since $R=2|D|$ is not admissible) so that for a fixed $k>0$ the minimum of (17) occurs for $\theta=\theta_{0}$, the maximum for $\theta=\theta_{0}+\pi$.

Let us now minimize (17) with respect to $k$ for $\theta=\theta_{0}$. The derivative with respect to $k$ of the part of (17) involving $k$ and $\theta$ becomes

$$
\begin{aligned}
p(2|D| & -R)^{p-2}\{(1-p)(R-|D|) \partial R / \partial k \\
& +[(2 p-3) R+2(2-p)|D|] \partial|D| / \partial k\} .
\end{aligned}
$$

We have $\partial R / \partial k=\left[R \partial|D| / \partial k-4|\alpha|^{2} k(2 / p-1)\right](R-|D|)^{-1}$. The case $R=|D|$ is admissible only if $p=1$, in which case $|p D|=|2 A|$; this is considered in the discussion of the second form for $1 \leqq p$ $<1+|\alpha|$. With $R \neq|D|$, (19) becomes $p(2|D|-R)^{p-2}(2-p)\left[(2|D|-R) \partial|D| / \partial k-4|\alpha|^{2}(1-p) p^{-1} k\right]$, which vanishes for $R=2|D|-4(1-p)|\alpha|^{2} k[p \partial|D| / \partial k]^{-1}$. With $\theta=\theta_{0}$ the value of $D$ is real and is $D=p^{-1}\left[p\left(1-|\alpha|^{2}\right)^{2 / p+1}-2 k|\alpha|^{2}\right]$. Only if $D>0$ will $k$ be admissible, so that $\partial|D| / \partial k=-2|\alpha|^{2} / p$. Thus $R=2|D|+2(1-p) k$ and we have

$$
2|D|+2(1-p) k=|D|-\left[|D|^{2}-4|A|^{2}(2 / p-1)\right]^{1 / 2} \text {. }
$$

With $|A|=k|\alpha|$ the only possibly valid solution of this equation is

$$
k=(p-1)\left(1-|\alpha|^{2}\right)^{2 / p+1}\left[(p-1)^{2}+|\alpha|^{2}\right]^{-1} .
$$

With this value of $k$ the value of $|b|$ in (16) is computed as $|b|$ $=|\alpha| /(p-1)$, and the value of $k$ in (20) is thus admissible if and only if $|\alpha| \leqq p-1$, in which case this value of $k$ and $\theta=\theta_{0}$ actually furnish the minimum, a relative minimum whose value is computed as $\phi_{2}(|\alpha|, p)$ as given in the statement of the theorem.

It has been shown that the minimum of $f(w)$ of the second form is $\phi_{2}(|\alpha|, p)$ if $1<1+|\alpha| \leqq p<2$, and is $2 \pi$ if $\alpha=0$. We may consistently define $\phi_{2}(0, p)=2 \pi$. Hence the minimum of $f(w)$ of the second form is $\phi_{2}(|\alpha|, p)$ if $1+|\alpha| \leqq p<2$.

Let us consider the second form of $f(w)$ for $p>2$. Here we set

$$
R=\left[|D|^{2}+4|A|^{2}(1-2 / p)\right]^{1 / 2}-|D| \text {. }
$$

The value of (15) now becomes

$$
\begin{aligned}
2 \pi \cdot 2^{1-p}\left[\left(1-|\alpha|^{2}\right)(p-2)\right]^{-1}(2|D|+R)^{p-1} \\
\cdot[(p-2)|D|+(p-1) R] .
\end{aligned}
$$

If $\alpha=0$ we have $D=1$ so that again (21) is a function of $|A|$ alone, its derivative with respect to $|A|$ vanishes only for $A=0$, and the 
minimum value is $2 \pi$. If $\alpha \neq 0$ we again set $A=k|\alpha| e^{i \theta}, \alpha=|\alpha| e^{i \theta_{0}}$. As in the case $p<2$, for fixed $k$ the minimum of (21) occurs for $\theta=\theta_{0}$, and with $\theta=\theta_{0}$ the minimizing value of $k$ is (20). With these values of $\theta$ and $k$ we have $|b|=|\alpha| /(p-1)$, so that $|b|<1$ for $p>2$. The values of $k$ and $\theta$ are admissible and the minimum for the second form with $p>2$ is $\phi_{2}(|\alpha|, p)$.

A combination of the results now permits us to state that the minimum of $f(w)$ of the second form is $\phi_{2}(|\alpha|, p)$ whenever $1+|\alpha|$ $\leqq p$.

We turn to a discussion of (17) when $1 \leqq p<1+|\alpha|$, in which case we must minimize (17) subject to the condition $|p D| \geqq|2 A|$. It has been shown that the relative minimum for fixed $k$ occurs for $\theta=\theta_{0}$. For a given $\theta$ the admissible minimum of (17) with respect to $k$ occurs for some value of $k$. For that value of $k$ the admissible minimum with respect to $\theta$ occurs either for $\theta=\theta_{0}$ or where $|p D|=|2 A|$. Among the values of (17) for $\theta=\theta_{0}$ the admissible minimum when $1 \leqq p<1+|\alpha|$ again occurs where $|p D|=|2 A|$. Hence in any event the admissible minimum of (17) occurs where $|p D|=|2 A|$, in which case (17) reduces to $2 \pi \cdot 2\left(1-|\alpha|^{2}\right)^{-1}|A|^{p}$, the minimum of $|A|$ occurs for $A=p \alpha(1-|\alpha|)\left(1-|\alpha|^{2}\right)^{2 / p}(2|\alpha|)^{-1}$, and the minimum value is $\phi_{3}(|\alpha|, p)$, which appears in (9).

The results thus far are the following, with $p=2$ again included. If $1 \leqq p \leqq 1+|\alpha|$ the minimum is $\phi_{1}(|\alpha|, p)$ for the first form, and $\phi_{3}(|\alpha|, p)$ for the second form. If $p>1+|\alpha|$ the minimum is $\phi_{3}(|\alpha|, p)$ for the first form and $\phi_{2}(|\alpha|, p)$ for the second form. We must now compare the two minima for each range of values of $p$. Let

I. We wish to show that $\phi_{1}(|\alpha|, p)<\phi_{3}(|\alpha|, p)$ if $1 \leqq p<1+|\alpha|$.

$$
\begin{aligned}
& x=p+|\alpha|^{2}-|\alpha|\left(|\alpha|^{2}-p^{2}+2 p\right)^{1 / 2}, \\
& y=(p-1)|\alpha|+\left(|\alpha|^{2}-p^{2}+2 p\right)^{1 / 2} .
\end{aligned}
$$

Then $\phi_{1}(|\alpha|, p)<\phi_{3}(|\alpha|, p)$ if

$$
(1+|\alpha|)\left(1+|\alpha|^{2}\right)^{-1}<p y^{2 / p-1}\left[\left(1+|\alpha|^{2}\right) x\right]^{-1 / p},
$$

which is in turn valid if

$$
\log \left[(1+|\alpha|)\left(1+|\alpha|^{2}\right)^{-1}\right]<\log \left\{p y^{2 / p-1}\left[\left(1+|\alpha|^{2}\right) x\right]^{-1 / p}\right\} .
$$

The two members are equal for $p=1+|\alpha|$; the inequality is therefore valid for $1 \leqq p<1+|\alpha|$ if the derivatives with respect to $p$ satisfy the reversed inequality, which becomes

$$
0>\log \left[\left(1+|\alpha|^{2}\right) x y^{-2}\right]
$$


since $x y-(p-2) x d y / d p-y d x / d p=0$. The last inequality is valid if

$$
\left(1+|\alpha|^{2}\right) x y^{-2}<1 .
$$

Now, $2\left(1+|\alpha|^{2}\right) x=x^{2}+y^{2}$ so that the last inequality is valid if $x^{2}-y^{2}<0$, which can easily be proved if $1 \leqq p<1+|\alpha|$. The desired inequality is thus proved.

II. We wish to show here that $\phi_{2}(|\alpha|, p)<\phi_{3}(|\alpha|, p)$ if $p>1$ $+|\alpha|$. If $\alpha=0$ it is easy to see that the inequality holds. If $\alpha \neq 0$ let $p-1=q$. Then $\phi_{2}(|\alpha|, p)<\phi_{3}(|\alpha|, p)$ if

$$
\left[\left(q^{2}+|\alpha|^{2}\right) /\left(2 q^{2}\right)\right]^{a}>[(1+|\alpha|) /(q+1)]^{q+1},
$$

which is valid if their logarithms are in the same relation:

$q\left[\log \left(q^{2}+|\alpha|^{2}\right)-\log \left(2 q^{2}\right)\right]>(q+1)[\log (1+|\alpha|)-\log (q+1)]$.

The two members are equal if $q=|\alpha|$; hence the inequality is valid for $q>|\alpha|$ if the derivatives are in the same relation, the resulting inequality becoming

$$
\begin{gathered}
\log \left(q^{2}+|\alpha|^{2}\right)-\log \left(2 q^{2}\right)-2|\alpha|^{2}\left(q^{2}+|\alpha|^{2}\right)^{-1}+1 \\
-\log (1+|\alpha|)+\log (q+1)>0 .
\end{gathered}
$$

The two members are again equal if $q=|\alpha|$; hence this inequality is valid for $q>|\alpha|$ if the derivative of the left member is positive, which statement may be expressed as

$$
P(q)=q^{5}+4|\alpha|^{2} q^{3}+2|\alpha|^{2} q^{2}-|\alpha|^{4} q-2|\alpha|^{4}>0 .
$$

The equation $P(q)=0$ has one variation in sign, hence by Descartes' rule of signs at most one positive root. But $P(0)=-2|\alpha|^{4}<0$, and $P(|\alpha|)=4|\alpha|^{5}>0$, so that there is a positive root, it lies between $q=0$ and $q=|\alpha|$, and for $q>|\alpha|$ the last inequality above is valid, and the proof is complete that $\phi_{2}(|\alpha|, p)<\phi_{3}(|\alpha|, p)$ if $q>|\alpha|$, or if $p>1+|\alpha|$.

The proof of the theorem is now complete.

In conclusion we mention that the minimizing function is unique except when $\alpha=0, p=1$. If $p<1+|\alpha|$, the minimizing function does not vanish for $|w| \leqq 1$. If $p>1+|\alpha|$, the minimizing function has a simple zero within the unit circle.

UNIVERSity of Wisconsin IN MilwaUkeE 